\title{
A simple synthesis of some pentafluoropropenyl derivatives of pyrimidine and purine based on addition-elimination reaction
}

\author{
Hanna Wójtowicz-Rajchel, Michał Migas and Henryk Koroniak* \\ Adam Mickiewicz University, Faculty of Chemistry, Grunwaldzka 6, 60-780 Poznań, \\ Poland \\ koroniak@amu.edu.pl
}

\section{Supporting Information}

\section{Contents:}

General information:

S1 - S2

Preparation and characterization data for compounds:

3a-3d, 4, 6a, b, e, 7, 9, and 11a-b S2 - S7

Preparation and characterization data for compound $\mathbf{1 2}$ S8

\section{General information}

${ }^{1} \mathrm{H}$ and ${ }^{19} \mathrm{~F}$ NMR spectra were obtained on $300 \mathrm{MHz}$ spectrometer in $\mathrm{CDCl}_{3}$ and DMSO-d $\mathrm{d}_{6}$ as solvents. TMS was the internal standard in ${ }^{1} \mathrm{H}$ NMR, $\mathrm{CFCl}_{3}$ was used as a reference for ${ }^{19} \mathrm{~F}$ NMR. Chemical shifts for ${ }^{1} \mathrm{H}$ NMR are reported in ppm downfield from TMS and for ${ }^{19} \mathrm{~F}$ NMR upfield 
from $\mathrm{CFCl}_{3}$. Chemical shifts are expressed in parts per million (ppm) and coupling constants in Hertz (Hz). Mass spectrometry was carried out in the electron impact (EI) and fast atom bombarding (FAB) mode. High resolution data were obtained using a peak matching technique. Elemental composition of the discussed ion was determined with an error of less than $10 \mathrm{ppm}$ in relation to perfluorokerosene at resolving power of 10000 . Wavenumbers of infrared spectra (IR) were given in $\mathrm{cm}^{-1}$ and only noteworthy absorption was listed. Melting points were uncorrected. Silica gel, 70-230 mesh, was used for column chromatography. All purchased starting materials were used without further purification. All solvents were of reagent grade quality and purchased commercially. Tetrahydrofuran was distilled from sodium hydride immediately before use. LDA was obtained from 2.0 $\mathrm{M} n$-BuLi and diisopropylamine according to a standard method. All reactions were carried out under an argon atmosphere. The reaction temperatures $-100^{\circ} \mathrm{C}$ and $-80^{\circ} \mathrm{C}$ were achieved by cooling with $\mathrm{N}_{2} /$ hexane bath

\section{Preparation and characterization data for compounds: 3a-3d, 4, 6a, b, e, 7, 9,}

\section{and 11a-b:}

To a stirred solution of LDA or $n$-BuLi (1.2-2.5 mmol) in anhydrous THF (20-50 mL) at $100^{\circ} \mathrm{C}$ or $-80^{\circ} \mathrm{C}$ a solution of compounds $2 \mathbf{a}-\mathbf{d}, 3 \mathbf{3 a}, \mathbf{5 a}-\mathbf{e}, \mathbf{8}$ or $\mathbf{1 0 a}-\mathbf{b}$ (1 mmol) was added dropwise. The reaction mixture was kept for 10-60 $\mathrm{min}$ at this temperature before a solution of hexafluoropropene (approximately $3 \mathrm{mmol}$ ) in $5 \mathrm{~mL}$ of THF was added using a Carrius tube. This mixture was kept at the above temperature for an additional 30 minutes and then allowed to warm to room temperature over a period of $3 \mathrm{~h}$. The solution was evaporated to dryness under reduced 
pressure and $10 \mathrm{~mL}$ of water was added. The aqueous solution was extracted with $\mathrm{CH}_{2} \mathrm{Cl}_{2}(2 \times 30$ $\mathrm{mL}$ ) and the combined extract dried over $\mathrm{Na}_{2} \mathrm{SO}_{4}$. Solvent was removed and the crude product was separated by column chromatography (silica gel, hexane, a gradient of hexane/ $\mathrm{CH}_{2} \mathrm{Cl}_{2}, \mathrm{CH}_{2} \mathrm{Cl}_{2}$ ) to give a mixture of $E / Z$ alkenes.

\section{2,4-Diethoxy-5-(perfluoroprop-1-enyl)pyrimidine (3a)}

Solidified colorless oil; yield $241 \mathrm{mg}(81 \%)$; Anal. calcd for $\mathrm{C}_{11} \mathrm{H}_{11} \mathrm{~N}_{2} \mathrm{O}_{2} \mathrm{~F}_{5}$ : C, 44.30; H, 3.72; N, 9.39. Found: C, 43.98; H, 3.52; N, 9.07; IR (film) v: 1720 m (CF=CF), 1682 m, 1645 m, 1599 s, $1555 \mathrm{~s}, 1345 \mathrm{~s}, 1204 \mathrm{~s}, 1149 \mathrm{~s} \mathrm{~cm}^{-1}$; MS (EI) $\mathrm{m} / z\left(\mathrm{M}^{+}\right)$298; HRMS: Calculated for $\mathrm{C}_{11} \mathrm{H}_{11} \mathrm{~N}_{2} \mathrm{O}_{2} \mathrm{~F}_{5}$ 298.07403, Found 298.07508; ${ }^{1} \mathrm{H}$ NMR $\left(\mathrm{CDCl}_{3}\right): \delta 1.35-1.55(\mathrm{~m}, 6 \mathrm{H}), 4.45-4.60(\mathrm{~m}, 4 \mathrm{H}), 8.25$ (Z isomer) and 8.35 (E isomer) $(\mathrm{s}, 1 \mathrm{H}) ;{ }^{19} \mathrm{~F}$ NMR $\left(\mathrm{CDCl}_{3}\right)$ isomer $E: \delta-66.6\left(\mathrm{dd},{ }^{3} \mathrm{~J}=11 \mathrm{~Hz},{ }^{4} \mathrm{~J}=22\right.$ $\mathrm{Hz}, 3 \mathrm{~F}),-135.0\left(\mathrm{dq},{ }^{3} \mathrm{~J}=141 \mathrm{~Hz},{ }^{4} \mathrm{~J}=22 \mathrm{~Hz}, 1 \mathrm{~F}\right),-164.5\left(\mathrm{dq},{ }^{3} \mathrm{~J}=141 \mathrm{~Hz},{ }^{3} \mathrm{~J}=11 \mathrm{~Hz}, 1 \mathrm{~F}\right)$, isomer $\mathrm{Z}$ : $\delta-66.5\left(\mathrm{dd},{ }^{3} \mathrm{~J}=8 \mathrm{~Hz},{ }^{4} \mathrm{~J}=14 \mathrm{~Hz}, 3 \mathrm{~F}\right),-110.8(\mathrm{~m}, 1 \mathrm{~F}),-149.9(\mathrm{~m}, 1 \mathrm{~F})$.

\section{2,4-Diisopropoxy-5-(perfluoroprop-1-enyl)pyrimidine (3b)}

Colorless oil; yield $261 \mathrm{mg}(80 \%)$; Anal. calcd for $\mathrm{C}_{13} \mathrm{H}_{15} \mathrm{~N}_{2} \mathrm{O}_{2} \mathrm{~F}_{5}$ : C, 47.86; H 4.63; N, 8.59. Found: C, 47.52; H, 4.50; N, 8.21; IR (film) v: 1717 m (CF=CF), 1670 m, 1635 m, 1597 s, 1554 s, $1360 \mathrm{~s}$, $1198 \mathrm{~s}, 1145 \mathrm{~s} \mathrm{~cm}^{-1}$; MS (EI) m/z (M+) 326; HRMS: Calculated for [M- $\left.\left(\mathrm{C}_{6} \mathrm{H}_{14}+\mathrm{H}_{2}\right)\right]^{+}: \mathrm{C}_{7} \mathrm{H}_{3} \mathrm{~N}_{2} \mathrm{O}_{2} \mathrm{~F}_{5}$ 242.01147, Found 242.01185; ${ }^{1} \mathrm{H}$ NMR $\left(\mathrm{CDCl}_{3}\right): \delta 1.36-1.43(\mathrm{~m}, 12 \mathrm{H}), 5.30$ and $5.47(\mathrm{~m}, 2 \mathrm{H})$, $8.23\left(\mathrm{Z}\right.$ isomer) and $8.33(\mathrm{E}$ isomer $)(\mathrm{s}, 1 \mathrm{H}) ;{ }^{19} \mathrm{~F} \mathrm{NMR}\left(\mathrm{CDCl}_{3}\right)$ isomer $E: \delta-65.7\left(\mathrm{dd},{ }^{3} \mathrm{~J}=11 \mathrm{~Hz},{ }^{4} \mathrm{~J}\right.$ $=22 \mathrm{~Hz}, 3 \mathrm{~F}),-134.2\left(\mathrm{dq},{ }^{3} \mathrm{~J}=140 \mathrm{~Hz},{ }^{4} \mathrm{~J}=22 \mathrm{~Hz}, 1 \mathrm{~F}\right),-163.5\left(\mathrm{dq},{ }^{3} \mathrm{~J}=140 \mathrm{~Hz},{ }^{3} \mathrm{~J}=11 \mathrm{~Hz}, 1 \mathrm{~F}\right)$, isomer $Z: \delta-65.6\left(\mathrm{dd},{ }^{3} \mathrm{~J}=8 \mathrm{~Hz},{ }^{4} \mathrm{~J}=13 \mathrm{~Hz}, 3 \mathrm{~F}\right),-109.4(\mathrm{~m}, 1 \mathrm{~F}),-149.4(\mathrm{~m}, 1 \mathrm{~F})$. 
Solidified colorless oil; yield $279 \mathrm{mg}$ (79\%); Anal. calcd for $\mathrm{C}_{15} \mathrm{H}_{19} \mathrm{~N}_{2} \mathrm{O}_{2} \mathrm{~F}_{5}$ : C, 50.85; H, 5.40; N, 7.91. Found: C, 50.46; H, 5.11; N, 7.53; IR (film) v: 1715 s (CF=CF), 1662 s, 1620 s, 1372 m, 1194 s, $1146 \mathrm{~s} \mathrm{~cm}^{-1}$; MS (EI) $\mathrm{m} / z\left(\mathrm{M}^{+}\right)$354; HRMS: Calculated for $\left[\mathrm{M}-\left(\mathrm{C}_{8} \mathrm{H}_{18}+\mathrm{H}_{2}\right)\right]^{+}: \mathrm{C}_{7} \mathrm{H}_{3} \mathrm{~N}_{2} \mathrm{O}_{2} \mathrm{~F}_{5}$ 242.01147, Found 242.00928; ${ }^{1} \mathrm{H}$ NMR $\left(\mathrm{CDCl}_{3}\right): \delta 1.60-1.65(\mathrm{~m}, 18 \mathrm{H}), 8.18$ (isomer Z) and 8.29

(isomer E) (s, $1 \mathrm{H}) ;{ }^{19} \mathrm{~F} \mathrm{NMR}\left(\mathrm{CDCl}_{3}\right)$ isomer $E: \delta-66.6\left(\mathrm{dd},{ }^{3} \mathrm{~J}=12 \mathrm{~Hz},{ }^{4} \mathrm{~J}=22 \mathrm{~Hz}, 3 \mathrm{~F}\right),-134.6(\mathrm{dq}$, $\left.{ }^{3} \mathrm{~J}=140 \mathrm{~Hz},{ }^{4} \mathrm{~J}=22 \mathrm{~Hz}, 1 \mathrm{~F}\right),-164.1\left(\mathrm{dq},{ }^{3} \mathrm{~J}=140 \mathrm{~Hz},{ }^{3} \mathrm{~J}=12 \mathrm{~Hz}, 1 \mathrm{~F}\right)$, isomer $Z: \delta-66.3\left(\mathrm{dd},{ }^{3} \mathrm{~J}=8\right.$ $\left.\mathrm{Hz},{ }^{4} \mathrm{~J}=14 \mathrm{~Hz}, 3 \mathrm{~F}\right),-109.7(\mathrm{~m}, 1 \mathrm{~F}),-151.2(\mathrm{~m}, 1 \mathrm{~F})$.

During storage compound $\mathbf{3 c}$ undergoes partly a decomposition to compound $\mathbf{1 2}$

\section{2,4-Dibenzyloxy-5-(perfluoroprop-1-enyl)pyrimidine (3d)}

Colorless solid, m.p. $48-51{ }^{\circ} \mathrm{C}$; yield $346 \mathrm{mg}$ (82\%); Anal. calcd for $\mathrm{C}_{21} \mathrm{H}_{15} \mathrm{~N}_{2} \mathrm{O}_{2} \mathrm{~F}_{5}$ : C, 59.72; $\mathrm{H}$, 3.58; N, 6.63. Found: C, 59.49; H, 3.49; N, 6.60; IR (KBr) v: 1720 m (CF=CF), 1685 m, 1648 m, 1598 s, 1553 s, 1435 s, 1361 s, 1207 s, 1145 s cm$^{-1}$; MS (EI) m/z (M+) 422; HRMS: Calculated for $\mathrm{C}_{21} \mathrm{H}_{15} \mathrm{~N}_{2} \mathrm{O}_{2} \mathrm{~F}_{5} 422.10538$, Found 422.10845; ${ }^{1} \mathrm{H} \mathrm{NMR}\left(\mathrm{CDCl}_{3}\right): \delta 5.46-5.50(\mathrm{~m}, 4 \mathrm{H}), 7.36-7.48$ $(\mathrm{m}, 10 \mathrm{H}), 8.28$ (isomer $\mathrm{Z}$ ) and 8.41 (isomer $\mathrm{E})(\mathrm{s}, 1 \mathrm{H}) ;{ }^{19} \mathrm{~F} \mathrm{NMR}\left(\mathrm{CDCl}_{3}\right)$ isomer $E: \delta-66.6\left(\mathrm{dd},{ }^{3} \mathrm{~J}=\right.$ $\left.11 \mathrm{~Hz},{ }^{4} \mathrm{~J}=22 \mathrm{~Hz}, 3 \mathrm{~F}\right),-135.1\left(\mathrm{dq},{ }^{3} \mathrm{~J}=141 \mathrm{~Hz},{ }^{4} \mathrm{~J}=22 \mathrm{~Hz}, 1 \mathrm{~F}\right),-164.0\left(\mathrm{dq},{ }^{3} \mathrm{~J}=141 \mathrm{~Hz},{ }^{3} \mathrm{~J}=11 \mathrm{~Hz}\right.$, 1F), isomer $Z: \delta-66.0\left(\mathrm{dd},{ }^{3} \mathrm{~J}=8 \mathrm{~Hz},{ }^{4} \mathrm{~J}=14 \mathrm{~Hz}, 3 \mathrm{~F}\right),-110.6(\mathrm{~m}, 1 \mathrm{~F}),-149.0(\mathrm{~m}, 1 \mathrm{~F})$.

\section{2,4-Diethoxy-5,6-bis(perfluoroprop-1-enyl)pyrimidine (4)}

Colorless oil, $184 \mathrm{mg}$ (43\%); Anal. calcd for $\mathrm{C}_{14} \mathrm{H}_{10} \mathrm{~N}_{2} \mathrm{O}_{2} \mathrm{~F}_{10}$ : C, 39.27; H, 2.36; N, 6.54. Found: C, 38.97; H, 2.62; N, 6.42; IR (film)v: $1731 \mathrm{w}(\mathrm{CF}=\mathrm{CF}), 1583 \mathrm{~s}, 1551 \mathrm{~s}, 1345 \mathrm{~s}, 1228 \mathrm{~s}, 1153 \mathrm{~s} \mathrm{~cm}^{-1}$; MS (EI) $m / z\left(\mathrm{M}^{+}\right)$428; HRMS: Calculated for $\mathrm{C}_{14} \mathrm{H}_{10} \mathrm{~N}_{2} \mathrm{O}_{2} \mathrm{~F}_{10}$ 428.05826, Found 428.05534; ${ }^{1} \mathrm{H}$ NMR $\left(\mathrm{CDCl}_{3}\right): \delta 1.30-1.50(\mathrm{~m}, 6 \mathrm{H}), 4.41-4.60(\mathrm{~m}, 4 \mathrm{H}) ;{ }^{19} \mathrm{~F}$ NMR $\left(\mathrm{CDCl}_{3}\right)$ isomer $E E: \delta$ from - 
66.9 to $67.2\left(\mathrm{dd},{ }^{3} \mathrm{~J}=10-12 \mathrm{~Hz},{ }^{4} \mathrm{~J}=20-22 \mathrm{~Hz}, 6 \mathrm{~F}\right),-132.1\left(\mathrm{dq},{ }^{3} \mathrm{~J}=139 \mathrm{~Hz},{ }^{4} \mathrm{~J}=22 \mathrm{~Hz}, 1 \mathrm{~F}\right),-$ $145.6\left(\mathrm{dq},{ }^{3} \mathrm{~J}=134 \mathrm{~Hz},{ }^{4} \mathrm{~J}=22 \mathrm{~Hz}, 1 \mathrm{~F}\right),-157.9\left(\mathrm{dq},{ }^{3} \mathrm{~J}=134 \mathrm{~Hz},{ }^{3} \mathrm{~J}=10 \mathrm{~Hz}, 1 \mathrm{~F}\right),-161.2\left(\mathrm{dq},{ }^{3} \mathrm{~J}=\right.$ $\left.139 \mathrm{~Hz},{ }^{3} \mathrm{~J}=11 \mathrm{~Hz}, 1 \mathrm{~F}\right)$, isomer $E Z: \delta$ from -66.9 to $-67.2\left(\mathrm{dd},{ }^{3} \mathrm{~J}=10-12 \mathrm{~Hz},{ }^{4} \mathrm{~J}=20-22 \mathrm{~Hz}, 3 \mathrm{~F}\right)$, $-67.7\left(\mathrm{dd},{ }^{3} \mathrm{~J}=6 \mathrm{~Hz},{ }^{4} \mathrm{~J}=12 \mathrm{~Hz}, 3 \mathrm{~F}\right),-111.4(\mathrm{~m}, 1 \mathrm{~F}),-146.1(\mathrm{~m}, 1 \mathrm{~F}),-145.2\left(\mathrm{dq},{ }^{3} \mathrm{~J}=135 \mathrm{~Hz},{ }^{4} \mathrm{~J}=21\right.$ $\mathrm{Hz}, 1 \mathrm{~F}),-156.7\left(\mathrm{dq},{ }^{3} \mathrm{~J}=135 \mathrm{~Hz},{ }^{3} \mathrm{~J}=10 \mathrm{~Hz}, 1 \mathrm{~F}\right)$.

\section{1,3-Dimethyl-6-(perfluoroprop-1-enyl)-5-fluorouracil (6a)}

Yellowish solid, m.p. 57-60 ${ }^{\circ} \mathrm{C}$; yield $170 \mathrm{mg}(59 \%)$; Anal. calcd for $\mathrm{C}_{9} \mathrm{H}_{6} \mathrm{~N}_{2} \mathrm{O}_{2} \mathrm{~F}_{6}$ : C, 37.52; $\mathrm{H}$, 2.10; N, 9.72. Found: C, 37.81; H, 2.29; N, 9.34; IR (film) v: $1726 \mathrm{~m}(\mathrm{CF}=\mathrm{CF}), 1678 \mathrm{~s}, 1656 \mathrm{~s}$, 1496 s, 1363 s, 1303 s, 1230 s, 1159 s, 748 s cm$^{-1}$; MS (EI) m/z (M+) 288; HRMS: Calculated for $\mathrm{C}_{9} \mathrm{H}_{6} \mathrm{~N}_{2} \mathrm{O}_{2} \mathrm{~F}_{6}$ 288.03336, Found 288.03117; ${ }^{1} \mathrm{H}$ NMR $\left(\mathrm{CDCl}_{3}\right): \delta 3.42$ and $3.43(\mathrm{~s}, 6 \mathrm{H}) ;{ }^{19} \mathrm{~F}$ NMR $\left(\mathrm{CDCl}_{3}\right)$ isomer $E: \delta-67.0\left(\mathrm{dd},{ }^{3} \mathrm{~J}=11 \mathrm{~Hz},{ }^{4} \mathrm{~J}=21 \mathrm{~Hz}, 3 \mathrm{~F}\right),-141.7\left(\mathrm{dqm},{ }^{3} \mathrm{~J}=143 \mathrm{~Hz},{ }^{4} \mathrm{~J}=21 \mathrm{~Hz}\right.$, $1 \mathrm{~F}),-148.1\left(\mathrm{dd},{ }^{5} \mathrm{~J}=17 \mathrm{~Hz},{ }^{4} \mathrm{~J}=6 \mathrm{~Hz}, 1 \mathrm{~F}\right),-152.7\left(\mathrm{ddq},{ }^{3} \mathrm{~J}=143 \mathrm{~Hz},{ }^{3} \mathrm{~J}=11 \mathrm{~Hz},{ }^{5} \mathrm{~J}=17 \mathrm{~Hz}, 1 \mathrm{~F}\right)$, isomer $Z: \delta-68.3(\mathrm{~m}, 3 \mathrm{~F}),-123.6(\mathrm{~m}, 1 \mathrm{~F}),-140.5(\mathrm{~m}, 1 \mathrm{~F}),-149.1(\mathrm{~m}, 1 \mathrm{~F})$.

\section{1,3-Dimethyl-6-(perfluoroprop-1-enyl)thymine (6b)}

Yellowish oil, yield (dependent on a temperature of the reaction) 71-134 mg (25-47\%); Anal. calcd for $\mathrm{C}_{10} \mathrm{H}_{9} \mathrm{~N}_{2} \mathrm{O}_{2} \mathrm{~F}_{5}: \mathrm{C}, 42.26 ; \mathrm{H}, 3.19 ; \mathrm{N}, 9.86$. Found: C, 41.88; H, 2.98; N, 9.56; IR (film)v: $1713 \mathrm{~s}$ $(\mathrm{CF}=\mathrm{CF}), 1669 \mathrm{~s}, 1472 \mathrm{~m}, 1372 \mathrm{~s}, 1128 \mathrm{~s}, 1159 \mathrm{~s} \mathrm{~cm}^{-1}$; MS (EI) $\mathrm{m} / z\left(\mathrm{M}^{+}\right)$284;

HRMS: Calculated for $\mathrm{C}_{10} \mathrm{H}_{9} \mathrm{O}_{2} \mathrm{~N}_{2} \mathrm{~F}_{5}$ 284.05841, Found 284.05779; ${ }^{1} \mathrm{H}$ NMR $\left(\mathrm{CDCl}_{3}\right): \delta 2.02-2.05$ $(\mathrm{m}, 3 \mathrm{H}), 3.40-3.42(\mathrm{~m}, 6 \mathrm{H}) ;{ }^{19} \mathrm{~F} \mathrm{NMR}\left(\mathrm{CDCl}_{3}\right)$ isomer $E: \delta-66.9\left(\mathrm{dd},{ }^{3} \mathrm{~J}=10 \mathrm{~Hz},{ }^{4} \mathrm{~J}=21 \mathrm{~Hz}, 3 \mathrm{~F}\right),-$ $136.1\left(\mathrm{dq},{ }^{3} \mathrm{~J}=147 \mathrm{~Hz},{ }^{4} \mathrm{~J}=21 \mathrm{~Hz}, 1 \mathrm{~F}\right),-157.4\left(\mathrm{dq},{ }^{3} \mathrm{~J}=147 \mathrm{~Hz},{ }^{3} \mathrm{~J}=10 \mathrm{~Hz}, 1 \mathrm{~F}\right)$, isomer $Z: \delta-68.0$ $\left(\mathrm{dd},{ }^{3} \mathrm{~J}=6 \mathrm{~Hz},{ }^{4} \mathrm{~J}=13 \mathrm{~Hz}, 3 \mathrm{~F}\right),-118.6(\mathrm{~m}, 1 \mathrm{~F}),-146.3(\mathrm{~m}, 1 \mathrm{~F})$. 


\section{6-[5-(Perfluoroprop-1-enyl)-hexahydro-1,3,5-trimethyl-2,6-dioxopyrimidyn-4-yl]-1,3,5- trimethylpyrimidine-2,4-dione (7)}

Solidified colorless oil, yield (dependent on a temperature of the reaction) 66-101 $\mathrm{mg}$ (15-23\%); Anal. calcd for $\mathrm{C}_{17} \mathrm{H}_{19} \mathrm{~N}_{4} \mathrm{O}_{4} \mathrm{~F}_{5}$ : C, 46.58; H, 4.37; N, 12.78. Found: C, 46.26; H, 4.49; N, 12.39; IR (KBr) v: $1705 \mathrm{~s}(\mathrm{CF}=\mathrm{CF}), 1682 \mathrm{~s}, 1653 \mathrm{~s}, 1480 \mathrm{~m}, 1363 \mathrm{~m}, 1221 \mathrm{~m}, 1145 \mathrm{~m} \mathrm{~cm}^{-1}$; MS (FAB) m/z $\left(\mathrm{M}^{+}+1\right)$ 439; HRMS: Calculated for $\mathrm{C}_{17} \mathrm{H}_{19} \mathrm{~N}_{4} \mathrm{O}_{4} \mathrm{~F}_{5}$ 438.13266, Found 438.13576; ${ }^{1} \mathrm{H}$ NMR $\left(\mathrm{CDCl}_{3}\right)$ : compound $7 \mathrm{a}: \delta 1.55(\mathrm{~s}, 3 \mathrm{H}), 1.80(\mathrm{~s}, 3 \mathrm{H}), 3.05,3.30,3.37$ and $3.63(\mathrm{~s}, 12 \mathrm{H}), 5.22(\mathrm{~s}, 1 \mathrm{H})$, compound 7b: $\delta 1.52(\mathrm{~s}, 3 \mathrm{H}), 2.20(\mathrm{~s}, 3 \mathrm{H}), 3.03,3.20,3.31$ and $3.40(\mathrm{~s}, 12 \mathrm{H}), 5.35(\mathrm{~s}, 1 \mathrm{H}) ;{ }^{19} \mathrm{~F}$ $\operatorname{NMR}\left(\mathrm{CDCl}_{3}\right): \delta-66.21\left(\mathrm{dd},{ }^{3} \mathrm{~J}=10 \mathrm{~Hz},{ }^{4} \mathrm{~J}=22 \mathrm{~Hz}, 2 \times 3 \mathrm{~F}\right),-146.8$ and $-147.4\left(\mathrm{dq},{ }^{3} \mathrm{~J}=137 \mathrm{~Hz},{ }^{4} \mathrm{~J}=\right.$ $22 \mathrm{~Hz}, 2 \times 1 \mathrm{~F}),-163.0$ and $-163.3\left(\mathrm{dq},{ }^{3} \mathrm{~J}=137 \mathrm{~Hz},{ }^{3} \mathrm{~J}=10 \mathrm{~Hz}, 2 \times 1 \mathrm{~F}\right)$.

\section{8-(Perfluoroprop-1-enyl)caffeine (9)}

Yellowish oil, $55 \mathrm{mg}$ (17\%); Anal. calcd for $\mathrm{C}_{11} \mathrm{H}_{9} \mathrm{~N}_{4} \mathrm{O}_{2} \mathrm{~F}_{5}$ : C, 40.75; H, 2.80; N, 17.28. Found: C, 40.90; H, 2.72; N, 17.36; IR (film) v: $1710 \mathrm{~s}(\mathrm{CF}=\mathrm{CF}), 1668, \mathrm{~s}, 1549 \mathrm{~m}, 1350 \mathrm{~m}, 1211 \mathrm{~m}, 1156 \mathrm{~m}$ $\mathrm{cm}^{-1}$; MS (EI) $\mathrm{m} / z\left(\mathrm{M}^{+}\right) 324$; HRMS: Calculated for $\mathrm{C}_{11} \mathrm{H}_{9} \mathrm{~N}_{4} \mathrm{O}_{2} \mathrm{~F}_{5} 324.06458$, Found 324.06302; ${ }^{1} \mathrm{H}$ NMR $\left(\mathrm{CDCl}_{3}\right.$ ): $\delta 3.42$ (isomer Z) and 3.43 (isomer E) (s, 3H), 3.58 (isomer Z) and 3.60 (isomer E) $(\mathrm{s}, 3 \mathrm{H}), 4.05$ (isomer Z) and 4.12 (isomer E) $\left(\mathrm{d},{ }^{\mathrm{Z}} \mathrm{J}_{\mathrm{F}-\mathrm{H}}=2 \mathrm{~Hz},{ }^{\mathrm{E}} \mathrm{J}_{\mathrm{F}-\mathrm{H}}=3 \mathrm{~Hz}, 3 \mathrm{H}\right) ;{ }^{19} \mathrm{~F}$ NMR $\left(\mathrm{CDCl}_{3}\right)$ isomer $E: \delta-66.6\left(\mathrm{dd},{ }^{3} \mathrm{~J}=11 \mathrm{~Hz},{ }^{4} \mathrm{~J}=22 \mathrm{~Hz}, 3 \mathrm{~F}\right),-145.2\left(\mathrm{ddq},{ }^{3} \mathrm{~J}=140 \mathrm{~Hz},{ }^{4} \mathrm{~J}=22 \mathrm{~Hz}, \mathrm{~J}_{\mathrm{F}-\mathrm{H}}=3 \mathrm{~Hz}\right.$, 1F), $-157.1\left(\mathrm{dq},{ }^{3} \mathrm{~J}=140 \mathrm{~Hz},{ }^{3} \mathrm{~J}=11 \mathrm{~Hz}, 1 \mathrm{~F}\right)$, isomer $Z: \delta-67.0\left(\mathrm{dd},{ }^{3} \mathrm{~J}=7 \mathrm{~Hz},{ }^{4} \mathrm{~J}=13 \mathrm{~Hz}, 3 \mathrm{~F}\right),-120.2$ (m, 1F), -141.3 (m, 1F). 
Compound was not isolated, ${ }^{19} \mathrm{~F}$ NMR yield was given using $m$-fluorotoluene as an internal standard.

${ }^{19} \mathrm{~F} \mathrm{NMR}\left(\mathrm{CDCl}_{3}\right)$ isomer $E: \delta-67.1\left(\mathrm{dd},{ }^{3} \mathrm{~J}=11 \mathrm{~Hz},{ }^{4} \mathrm{~J}=21 \mathrm{~Hz}, 3 \mathrm{~F}\right),-113.4\left(\mathrm{dq},{ }^{3} \mathrm{~J}=134 \mathrm{~Hz},{ }^{4} \mathrm{~J}=21\right.$ $\mathrm{Hz}, 1 \mathrm{~F}),-160.8\left(\mathrm{dq},{ }^{3} \mathrm{~J}=134 \mathrm{~Hz},{ }^{3} \mathrm{~J}=11 \mathrm{~Hz}, 1 \mathrm{~F}\right)$, isomer $Z: \delta-67.0($ broad $\mathrm{m}, 3 \mathrm{~F}),-94.2(\mathrm{~m}, 1 \mathrm{~F}),-$ $148.4(\mathrm{~m}, 1 \mathrm{~F})$.

\section{2'3'5'Tribenzoyl-1-(perfluoroprop-1-enyl)inosine (11b)}

Cream-yellow crystals, m.p. $126-130{ }^{\circ} \mathrm{C}$; yield $518 \mathrm{mg}(73 \%)$; Anal. calcd for $\mathrm{C}_{34} \mathrm{H}_{23} \mathrm{~N}_{4} \mathrm{O}_{8} \mathrm{~F}_{5}$ : C, 57.47; H, 3.33; N, 7.88. Found: C, 57.12; H, 3.52; N, 7.62; IR (KBr) v: 1729 br s, 1373 m, 1269 s, $1213 \mathrm{~m}, 1122 \mathrm{~m}, 710 \mathrm{~m} \mathrm{~cm}^{-1}$; MS (FAB) m/z $\left(\mathrm{M}^{+}+1\right)$ 711, (EI) 445; HRMS: Calculated for [M$\left.\left(\mathrm{C}_{8} \mathrm{H}_{2} \mathrm{~N}_{4} \mathrm{OF}_{5}\right)\right]^{+}: \mathrm{C}_{26} \mathrm{H}_{21} \mathrm{O}_{7} 445.12872$, Found 445.12713; ${ }^{1} \mathrm{H} \mathrm{NMR}\left(\mathrm{CDCl}_{3}\right): \delta 4.68,4.83,4,94$ and $6.15(\mathrm{~m}, 4 \mathrm{H}), 6.30(\mathrm{~m}, 2 \mathrm{H}), 7.35-7.62$ and $7.91-8.11(\mathrm{~m}, 16 \mathrm{H}), 8.05(\mathrm{~m}, 1 \mathrm{H}) ;{ }^{19} \mathrm{~F} \mathrm{NMR}\left(\mathrm{CDCl}_{3}\right)$ isomer $E: \delta-67.2\left(\mathrm{dd},{ }^{3} \mathrm{~J}=11 \mathrm{~Hz},{ }^{4} \mathrm{~J}=21 \mathrm{~Hz}, 3 \mathrm{~F}\right),-113.6\left(\mathrm{dq},{ }^{3} \mathrm{~J}=133 \mathrm{~Hz},{ }^{4} \mathrm{~J}=21 \mathrm{~Hz}, 1 \mathrm{~F}\right),-160.8$ $\left(\mathrm{dq},{ }^{3} \mathrm{~J}=133 \mathrm{~Hz},{ }^{3} \mathrm{~J}=11 \mathrm{~Hz}, 1 \mathrm{~F}\right)$, isomer $Z: \delta-67.0(\operatorname{broad} \mathrm{m}, 3 \mathrm{~F}),-94.2(\mathrm{~m}, 1 \mathrm{~F}),-148.6(\mathrm{~m}, 1 \mathrm{~F})$.

\section{Preparation and characterization data for compound 12}

\section{5-(perfluoroprop-1-enyl)uracil (12)}

To a solution of $3 \mathrm{c}(354 \mathrm{mg}, 1 \mathrm{mmol})$ in $10 \mathrm{~mL}$ of ether, $2 \mathrm{ml}$ of $5 \%$ solution of $\mathrm{HCl}$ in methanol was added at room temperature and the reaction mixture was stirred for 30 minutes. Then, $10 \mathrm{~mL}$ of water was added and the organic layer was separated. The aqueous layer was extracted 
with ether $(2 \times 10 \mathrm{~mL})$, the combined ether extract was washed with saturated sodium bicarbonate, water, dried over $\mathrm{Na}_{2} \mathrm{SO}_{4}$ and concentrated in vacuo to give 12: white crystals, m.p. $188-192{ }^{\circ} \mathrm{C}$; yield $224 \mathrm{mg}(93 \%)$, Anal. calcd for $\mathrm{C}_{7} \mathrm{H}_{3} \mathrm{~N}_{2} \mathrm{O}_{2} \mathrm{~F}_{5}: \mathrm{C}, 34.73 ; \mathrm{H}, 1.25 ; \mathrm{N}, 11.57$. Found: $\mathrm{C}, 34.87 ; \mathrm{H}$, 1,52; N, 11.32; IR (KBr) v: 3244 m, 3197 m, 1724 s, 1688 s, 1382 m, 1296 m, 1205 m, $1147 \mathrm{~m} \mathrm{~cm}^{-1}$; MS (EI) $m / z\left(\mathrm{M}^{+}\right)$242; HRMS: Calculated for $\mathrm{C}_{7} \mathrm{H}_{3} \mathrm{~N}_{2} \mathrm{O}_{2} \mathrm{~F}_{5}$ 242.01147, Found 242.00953; ${ }^{1} \mathrm{H}$ NMR (DMSO-d $\mathrm{d}_{6}$ ): $\delta 7.91$ (isomer Z) and 8.14 (isomer E) (s, 1H), 11.63 (broad s, 2H); ${ }^{19} \mathrm{~F}$ NMR (DMSO$\left.\mathrm{d}_{6}\right): \mathrm{E} / \mathrm{Z}$ ratio $=73 / 27$, isomer $E: \delta-65.9\left(\mathrm{dd},{ }^{3} \mathrm{~J}=12 \mathrm{~Hz},{ }^{4} \mathrm{~J}=22 \mathrm{~Hz}, 3 \mathrm{~F}\right),-131.2\left(\mathrm{dq},{ }^{3} \mathrm{~J}=141 \mathrm{~Hz},{ }^{4} \mathrm{~J}\right.$ $=22 \mathrm{~Hz}, 1 \mathrm{~F}),-165.9\left(\mathrm{dq},{ }^{3} \mathrm{~J}=141 \mathrm{~Hz},{ }^{3} \mathrm{~J}=12 \mathrm{~Hz}, 1 \mathrm{~F}\right)$, isomer $Z: \delta-65.7\left(\mathrm{dd},{ }^{3} \mathrm{~J}=8 \mathrm{~Hz},{ }^{4} \mathrm{~J}=14 \mathrm{~Hz}\right.$, 3F), $-106.5(\mathrm{~m}, 1 \mathrm{~F}),-152.7(\mathrm{~m}, 1 \mathrm{~F})$. 